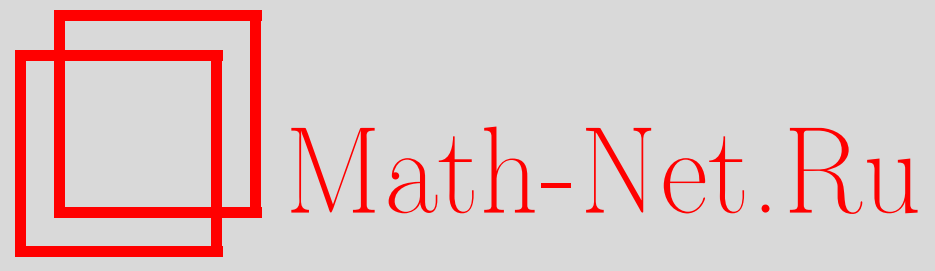

Я. А. Шарифов, Задача оптимального управления для систем с импульсными воздействиями при нелокальных краевых условиях, Вестн. Сам. гос. техн. ун-та. Сер. Физ.-мат. науки, 2013, выпуск 4(), 34-45

DOI: https://doi.org/10.14498/vsgtu1134

Использование Общероссийского математического портала MathNet.Ru подразумевает, что вы прочитали и согласны с пользовательским соглашением

http://www.mathnet.ru/rus/agreement

Параметры загрузки:

IP : 3.95 .254 .165

26 апреля 2023 г., 13:18:13

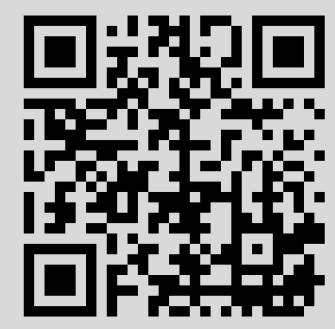




\title{
ЗАДАЧА ОПТИМАЛЬНОГО УПРАВЛЕНИЯ ДЛЯ СИСТЕМ С ИМПУЛЬСНЫМИ ВОЗДЕЙСТВИЯМИ ПРИ НЕЛОКАЛЬНЫХ КРАЕВЫХ УСЛОВИЯХ
}

\author{
Я. А. Шарифов \\ 1 Бакинский государственный университет, \\ Азербайджан, AZ-1073/1, Баку, ул. Академика Захид Халилова, 23. \\ 2 Институт кибернетики им. ак. А. Гусейнова НАН Азербайджана, \\ Азербайджан, AZ1141, Баку, ул. Ф. Агаева, 9. \\ E-mail: sharifov22@rambler.ru
}

\begin{abstract}
Исследуется задача оптимального управления, где состояние управляемой системы описывается дифференциальными уравнениями с импульсными воздействиями при нелокальных краевых условиях. С помощъю принципа сжатых отображений доказаны существование и единственность решения нелокальной краевой задачи при импульсных воздействиях при биксированных допустимых управлениях. При некоторых ограничениях на исходные данные задачи вычислен градиент функиионала и выведены необходимые условия оптимальности.
\end{abstract}

Ключевые слова: нелокальные краевые условия, импульсные системь, условия оптимальности, градиент функиионала, существование и единственность решения.

Введение. Дифференциальные уравнения с импульсным воздействием моделируют поведение эволюционирующих во времени процессов разнообразной природы, которые могут почти мгновенно изменять свое состояние. Такие уравнения имеют разрывные решения с разрывами первого рода в фиксированные или нефиксированные моменты времени [1]. В [2, стр. 10, 16] приведены конкретные примеры из теории электрических колебаний и часов, математические модели которых описываются дифференциальными уравнениями с импульсными воздействиями. Такие дифференциальные уравнения подробно изучены в монографиях [1-4]. Однако в последние годы интенсивно исследуются дифференциальные уравнения с импульсными воздействиями при нелокальных краевых условиях (см. напр., [4-8] и ссылки в них). В этих работах отмечается существование многочисленных процессов физики, техники, экологии, механики и др., математические модели которых описываются дифференциальными уравнениями с импульсными воздействиями при нелокальных краевых условиях и доказаны теоремы существования и единственности решения соответствующих краевых задач. В указанных работах нелокальность краевых условий в основном имеет вид

$$
x(0)+g(x)=x_{0},
$$

где $g(x)$ - линейный оператор, действующий из пространства кусочных абсолютно непрерывных функций в пространство $\mathbb{R}^{n}$. Такие краевые условия не охватывают даже простые случаи, например, когда одна часть граничных условий задана в начальной точке, а другая часть - в конечной точке.

Ягуб Амияр Шарифов (к.ф.-м.н., доц.), доцент, факультет прикладной математики и кибернетики, каф. математических методов прикладного анализа ${ }^{1}$; старший научный сотрудник, лаб. численных методов оптимального управления ${ }^{2}$. 
В монографии [9] приведены примеры из практики, в которых нелокальные граничные условия заданы в различных точках, т. е. имеют вид

$$
\sum_{k=0}^{m} A_{k} x\left(t_{k}\right)=C \text {. }
$$

Очевидно, что такие нелокальные краевые условия более общие, чем выше отмеченные, так как содержат (1) как частный случай. Действительно, при $A_{0}=E(E-$ единичная матрица) получаются условия вида $(1)$.

В настоящей работе исследуется задача оптимального управления системой, состояние которой описывается дифференциальными уравнениями с импульсными воздействиями при нелокальных краевых условиях. Исследованы вопросы существования и единственности решений краевой задачи, найдены достаточные условия для дифференцируемости критерия качества, получена формула его градиента и установлены необходимые условия оптимальности в форме вариационных неравенств.

1. Постановка задачи. Рассмотрим следующую нелокальную краевую задачу при импульсных воздействиях:

$$
\begin{gathered}
\frac{d x}{d t}=f(t, x, u(t)), \quad 0 \leqslant t \leqslant T, \quad t \neq t_{i}, \\
A x(0)+B x(T)=C, \\
\Delta x\left(t_{i}\right)=I_{i}\left(x\left(t_{i}\right), v_{i}\right), \quad i=1,2, \ldots, p, 0<t_{1}<t_{2}<\cdots<t_{p}<T, \\
(u(\cdot),[v]) \in U \times \Pi^{p}=\left\{u(t) \in L_{2}^{r}[0, T]: u(t) \in V,\right. \\
\text { п.в. } \left.t \in[0, T], v_{i} \in \Pi, i=1,2, \ldots, p .\right\},
\end{gathered}
$$

где $x(t) \in \mathbb{R}^{n} ; f(t, x, u)$ - n-мерная непрерывная функция; $A, B \in \mathbb{R}^{n \times n}$, $C \in \mathbb{R}^{n \times 1}$ - заданные постоянные матрицы; $\Delta x\left(t_{i}\right)=x\left(t_{i}^{+}\right)-x\left(t_{i}^{-}\right) ; I_{i}(x, v)$ некоторые заданные функции; $(u,[v])$ - управляющие параметры; $V \in \mathbb{R}^{r}$ и $\Pi \in \mathbb{R}^{m}$ - ограниченные выпуклые множества.

Требуется на решениях краевой задачи (2)-(5) минимизировать функционал

$$
J(u,[v])=\Phi(x(0), x(T)),
$$

где $\Phi(x, y)$ - заданная скалярная функция.

Под решением краевой задачи (2)-(4), соответствующей фиксированному управляющему параметру $(u(\cdot),[v]) \in U \times \Pi^{p}$, будем понимать функцию $x(t):[0, T] \rightarrow \mathbb{R}^{n}$, абсолютно непрерывную на $[0, T], t \neq t_{i}$ и непрерывную слева при $t=t_{i}$, для которой существует конечный правый предел $x\left(t_{i}^{+}\right)$при $i=1,2, \ldots, p$. Пространство таких функций обозначим $P C\left([0, T], \mathbb{R}^{n}\right)$. Очевидно, такое пространство является банаховым с нормой $\|x\|_{P C}=\max _{t \in[0, T]}|x(t)|$, где $|\cdot|$ - является нормой в $\mathbb{R}^{n}$.

Допустимый процесс $\{(u(t),[v]), x(t ; u(t),[v])\}$, являющийся решением задачи (2)-(6), т. е. доставляющий минимум функционалу (6) при ограничениях $(2)-(5)$, будем называть оптимальным процессом, а $(u(t),[v])$ - оптимальным управлением, где через $x(t ; u(t),[v])$ обозначено решение краевой задачи $(2)-$ $(4)$, соответствующее допустимому управлению $(u(t),[v])$. 
2. Существование решений краевой задачи (2)-(4). Рассмотрим следующие условия:

1) $\operatorname{det}(A+B) \neq 0$;

2) $f:[0, T] \times \mathbb{R}^{n} \times \mathbb{R}^{r} \rightarrow \mathbb{R}^{n}, I_{i}: \mathbb{R}^{n} \times \mathbb{R}^{m} \rightarrow \mathbb{R}^{n}, i=1,2, \ldots, p$ - непрерывные функции; существуют постоянные $K>0, L_{i}>0, i=1,2, \ldots, p$, такие, что

$$
\begin{gathered}
|f(t, x, u)-f(t, y, u)| \leqslant K|x-y|, \quad t \in[0, T], \quad x, y \in \mathbb{R}^{n}, \\
\left|I_{i}(x, v)-I_{i}(y, v)\right| \leqslant L_{i}|x-y|, \quad x, y \in \mathbb{R}^{n}
\end{gathered}
$$

3)

$$
L=S\left[K T+\sum_{i=1}^{p} L_{i}\right]<1
$$

где $S=\max \left\{\left\|(A+B)^{-1} A\right\|,\left\|(A+B)^{-1} B\right\|\right\}$.

Теорема 1. Пусть выполняется условие 1). Тогда $x(t) \in P C\left([0, T], \mathbb{R}^{n}\right)$ является абсолютно непрерывным решением краевой задачи (2)-(4) тогда и только тогда, когда

$$
x(t)=(A+B)^{-1} C+\int_{0}^{T} K(t, \tau) f(\tau, x(\tau), u(\tau)) d \tau+\sum_{i=1}^{p} K\left(t, t_{i}\right) I_{i}\left(x\left(t_{i}\right), v_{i}\right),
$$

где

$$
K(t, \tau)=\left\{\begin{aligned}
(A+B)^{-1} A, & 0 \leqslant \tau \leqslant t \\
-(A+B)^{-1} B, & t \leqslant \tau \leqslant T
\end{aligned}\right.
$$

Доказ ательство. Если функция $x(t)$ является решением дифференциального уравнения $(2)$, то для $t \in\left(t_{j}, t_{j+1}\right)$

$$
\begin{aligned}
& \int_{0}^{t} f(s, x(s), u(s)) d s=\int_{0}^{t} x^{\prime}(s) d s= \\
& =\left[x\left(t_{1}^{-}\right)-x\left(0^{+}\right)\right]+\left[x\left(t_{2}^{-}\right)-x\left(t_{1}^{+}\right)\right]+\cdots+\left[x\left(t^{-}\right)-x\left(t_{j}^{+}\right)\right]= \\
& =-x(0)-\left[x\left(t_{1}^{+}\right)-x\left(t_{1}^{-}\right)\right]-\left[x\left(t_{2}^{+}\right)-x\left(t_{2}^{-}\right)\right]-\cdots-\left[x\left(t_{j}^{+}\right)-x\left(t_{j}^{-}\right)\right]+x(t) .
\end{aligned}
$$

Отсюда

$$
x(t)=x(0)+\int_{0}^{t} f(s, x(s), u(s)) d s+\sum_{0<t_{j}<t} \Delta x\left(t_{j}\right),
$$

где $x(0) \in \mathbb{R}^{n}$ - пока произвольный постоянный вектор. Для определения $x(0)$ потребуем, чтобы функция, определяемая равенством (8), удовлетворяла условию (3):

$$
(A+B) x(0)=C-B \int_{0}^{T} f(t, x(t), u(t)) d t-B \sum_{0<t_{j}<T} \Delta x\left(t_{j}\right) .
$$


Taк как $\operatorname{det}(A+B) \neq 0$,

$x(0)=(A+B)^{-1} C-(A+B)^{-1} B \int_{0}^{T} f(t, x(t), u(t)) d t-(A+B)^{-1} B \sum_{0<t_{j}<T} \Delta x\left(t_{j}\right)$.

Теперь в (8) учтём значение $x(0)$, определяемое равенством (9):

$$
\begin{aligned}
x(t) & =(A+B)^{-1} C-(A+B)^{-1} B \int_{0}^{T} f(t, x(t), u(t)) d t- \\
& -(A+B)^{-1} B \sum_{0<t_{i}<T} \Delta x\left(t_{i}\right)+\int_{0}^{t} f(s, x(s), u(s)) d s+\sum_{0<t_{i}<t} \Delta x\left(t_{i}\right)= \\
& =(A+B)^{-1} C+\int_{0}^{T} K(t, \tau) f(\tau, x(\tau), u(\tau)) d \tau+\sum_{i=1}^{p} K\left(t, t_{i}\right) I_{i}\left(x\left(t_{i}\right), v_{i}\right) .
\end{aligned}
$$

Таким образом, показано, что краевую задачу (2)-(4) можно представить в виде интегрального уравнения (7). Непосредственной проверкой показывается, что решение интегрального уравнения (7) также удовлетворяет краевой задаче (2)-(4).

Определим оператор $P: P C\left([0, T], \mathbb{R}^{n}\right) \rightarrow P C\left([0, T], \mathbb{R}^{n}\right)$ по правилу

$$
(P x)(t)=(A+B)^{-1} C+\int_{0}^{T} K(t, \tau) f(\tau, x(\tau), u(\tau)) d \tau+\sum_{i=1}^{p} K\left(t, t_{i}\right) I_{i}\left(x\left(t_{i}\right), v_{i}\right) .
$$

Теорема 2. Пусть выполняются условия 1)-3). Тогда для любого $C \in \mathbb{R}^{n}$ $u(u(\cdot),[v]) \in U \times \Pi^{p}$ краевая задача (2)-(4) имеет единственное решение, которое удовлетворяет равенству

$$
x(t)=(A+B)^{-1} C+\int_{0}^{T} K(t, \tau) f(\tau, x(\tau), u(\tau)) d \tau+\sum_{i=1}^{p} K\left(t, t_{i}\right) I_{i}\left(x\left(t_{i}\right), v_{i}\right) .
$$

Доказа тельст во. Пусть $C \in \mathbb{R}^{n}$ и $(u(t),[v]) \in U \times \Pi^{p}$ - фиксированы. Рассмотрим отображение $P: P C\left([0, T], \mathbb{R}^{n}\right) \rightarrow P C\left([0, T], \mathbb{R}^{n}\right)$, определяемое равенством (10). Тогда для любых $\omega, w \in P C\left([0, T], \mathbb{R}^{n}\right)$ имеем оценку

$$
\begin{gathered}
|(P \omega)(t)-(P w)(t)| \leqslant \int_{0}^{T}|K(t, s)| \cdot|f(s, \omega(s), u(s))-f(s, w(s), u(s))| d s+ \\
\quad+\sum_{i=1}^{p}\left|K\left(t, t_{i}\right)\right| \cdot\left|I_{i}\left(\omega\left(t_{i}\right), v_{i}\right)-I_{i}\left(w\left(t_{i}\right) v_{i}\right)\right| \leqslant \\
\leqslant S\left[K \int_{0}^{T}|\omega(s)-w(s)| d s+\sum_{i=1}^{p} L_{i} \cdot\left|\omega\left(t_{i}\right)-w\left(t_{i}\right)\right|\right] \leqslant \\
\leqslant S\left[K T+\sum_{i=1}^{p} L_{i}\right]\|\omega(t)-w(t)\|_{P C}, \quad t \in[0, T]
\end{gathered}
$$


или

$$
\|P v-P w\|_{P C} \leqslant L\|\omega-w\|_{P C} .
$$

Полученная оценка показывает, что оператор $P$ является сжимающим на $P C\left([0, T], \mathbb{R}^{n}\right)$. Поэтому, согласно принципу сжимающих операторов, оператор $P$, определяемый равенством (10), имеет единственную неподвижную точку в $P C\left([0, T], \mathbb{R}^{n}\right)$. Значит, интегральное уравнение (7) или краевая задача (2)-(4) имеет единственное решение.

ЗАмечание 1 . Теорема 2 содержит в себе различные частные случаи. Например, если $I_{i}(x, v)=0, i=0,1, \ldots, p$, то получается система дифференциальных уравнений без импульсных воздействий. Тогда предположение 3) превращается в условие

$$
K T S<1,
$$

которое является достаточным условием существования и единственности решения краевой задачи

$$
\dot{x}=f(t, x, u), \quad A x(0)+B x(T)=C .
$$

3. Градиент в задаче оптимального управления (2)-(5). Нетрудно показать, что при сделанных предположениях 1)-3) всякое решение краевой задачи (2)-(4) ограничено. Действительно, в силу ограниченности множества допустимых управлений из (11) имеем

$$
\begin{aligned}
x(t)=(A+B)^{-1} C & +\int_{0}^{T} K(t, \tau) f(\tau, 0, u(\tau)) d \tau+\sum_{i=1}^{p} K\left(t, t_{i}\right) I_{i}\left(0, v_{i}\right)+ \\
+\int_{0}^{T} K(t, \tau)[f(\tau, x(\tau), u(\tau))-f(\tau, 0, u(\tau))] d \tau+ & \\
& +\sum_{i=1}^{p} K\left(t, t_{i}\right)\left[I_{i}\left(x\left(t_{i}\right), v_{i}\right)-I_{i}\left(0, v_{i}\right)\right] .
\end{aligned}
$$

Отсюда

$$
(1-L)|x(t)| \leqslant S\left[l T+\sum_{i=1}^{p} l_{i}\right]+\left\|(A+B)^{-1} C\right\|,
$$

где

$$
l=\max _{t \in[0, T], u \in V}|f(t, 0, u)|, \quad l_{i}=\max _{v_{i} \in \Pi}\left|I_{i}\left(0, v_{i}\right)\right| .
$$

Таким образом, из последнего имеем

$$
|x| \leqslant(1-L)^{-1}\left\{S\left[l T+\sum_{i=1}^{p} l_{i}\right]+\left\|(A+B)^{-1} C\right\|\right\} \equiv R .
$$

Сформулируем теперь некоторые дополнительные условия на $f(t, x, u)$, $I_{i}(x, v), i=1,2, \ldots, p, \Phi(x, y)$, которые предполагаются выполненными для всех $|x| \leqslant R, u \in V, v_{i} \in \Pi, 0 \leqslant t \leqslant T$. 
4) Производные функции $f(t, x, u)$ относительно $u$ ограничены, т. е. для любого $\bar{u} \in \mathbb{R}^{r}$

$$
\left|f_{u}(t, x, u) \bar{u}\right| \leqslant K_{1}|\bar{u}|
$$

5) Производные $f(t, x, u)$ по $x$ и $u$ удовлетворяют условиям Липшица:

$$
\left|f(t, x+\bar{x}, u+\bar{u})-f(t, x, u)-f_{x}(t, x, u) \bar{x}-f_{u}(t, x, u) \bar{u}\right| \leqslant K_{2}|\bar{x}|^{2}+K_{3}|\bar{u}|^{2} .
$$

6) Производные $I_{i}(x, v), i=0,1, \ldots, p$, по $v$ ограничены:

$$
\left|I_{i v}(x, v) \bar{v}\right| \leqslant L_{i}^{(1)}|\bar{v}|
$$

7) Производные $I_{i}(x, v), i=1,2, \ldots, p$, по $x$ и $v$ удовлетворяют условиям Липшица:

$$
\left|I_{i}(x+\bar{x}, v+\bar{v})-I_{i}(x, v)-I_{i x}(x, v) \bar{x}-I_{i v}(x, v)\right| \leqslant L_{i}^{(2)}|\bar{x}|^{2}+L_{i}^{(3)}|v|^{2}
$$

8) Функция $\Phi(x, y)$ имеет ограниченные первые производные и эти производные удовлетворяют условию Липшица:

$$
\left|\Phi_{x}(x, y)\right| \leqslant K_{4} ; \quad\left|\Phi_{y}(x, y)\right| \leqslant K_{5} ;
$$

$\left|\Phi(x+\bar{x}, y+\bar{y})-\Phi(x, y)-\left\langle\Phi_{x}(x, y), \bar{x}\right\rangle-\left\langle\Phi_{y}(x, y), \bar{y}\right\rangle\right| \leqslant K_{6}|\bar{x}|^{2}+K_{7}|\bar{y}|^{2}$.

Лемма 1. Пусть выполняются условия 1)-4) u 6), a $(u(t),[v], x(t)) u$ $(u(t)+\bar{u}(t),[v+\bar{v}], x(t)+\bar{x}(t))-$ два решения краевой задачи $(2)-(5)$. Тогда

$$
|\bar{x}(t)| \leqslant c_{1}(\|\bar{u}\|+\|[v]\|)
$$

где $c_{1}=(1-L)^{-1} S \max \left[K_{1} \sqrt{T}, \max L_{i}^{(1)} \sqrt{p}\right]$.

Д о каз а т ель ст в о. Очевидно, $\bar{x}(t)$ является решением следующего интегрального уравнения:

$$
\begin{aligned}
\bar{x}(t)=\int_{0}^{T} K(t, \tau)[f(\tau, & x(\tau)+\bar{x}(\tau), u(\tau)+\bar{u}(\tau))-f(\tau, x(\tau), u(\tau))] d \tau+ \\
& +\sum_{i=1}^{p} K\left(t, t_{i}\right)\left[I_{i}\left(x\left(t_{i}\right)+\bar{x}\left(t_{i}\right), v_{i}+\bar{v}_{i}\right)-I_{i}\left(x\left(t_{i}\right), v_{i}\right)\right]
\end{aligned}
$$

Учитывая условия 2), 4) и 6), находим

$$
|\bar{x}(t)| \leqslant S\left[\int_{0}^{T}\left(K|\bar{x}(t)|+K_{1}|\bar{u}(t)|\right) d t\right]+S\left[\sum_{i=1}^{p}\left(L_{i}\left|\bar{x}\left(t_{i}\right)\right|+L_{i}^{(1)}\left|\bar{v}_{i}\right|\right)\right] .
$$


Отсюда легко можно получить оценку

$$
|\bar{x}(t)| \leqslant(1-L)^{-1} S\left[K_{1} \sqrt{T}\|\bar{u}\|+\max L_{i}^{(1)} \sqrt{p}\left(\sum_{i=1}^{p}\left|\bar{v}_{i}\right|^{2}\right)^{1 / 2}\right] .
$$

Таким образом, из последнего соотношения имеем

$$
|\bar{x}(t)| \leqslant(1-L)^{-1} S \max \left[K_{1} \sqrt{T}, \max L_{i}^{(1)} \sqrt{p}\right](\|\bar{u}\|+\|[\bar{v}]\|) .
$$

Рассмотрим системы уравнений в вариациях:

$$
\begin{gathered}
\frac{d z}{d t}=f_{x}(t, x(t), u(t)) z(t)+f_{u}(t, x(t), u(t)) \bar{u}(t), \quad 0 \leqslant t \leqslant T, \quad t \neq t_{i}, \\
A z(0)+B z(T)=0, \\
\Delta z\left(t_{i}\right)=I_{i x}\left(x\left(t_{i}\right), v_{i}\right) z\left(t_{i}\right)+I_{i v_{i}}\left(x\left(t_{i}\right), v_{i}\right) \bar{v}_{i}, \quad i=1,2, \ldots, p \\
0=t_{0}<t_{1}<t_{2}<\cdots<t_{p}<t_{p+1}=T .
\end{gathered}
$$

ЛЕмма 2. Пусть выполняются условия 1)-7); $(\bar{u}(t),[\bar{v}], \bar{x}(t))-$ те же, что и в лемме 1, a $z(t)$ - решение уравнения в вариациях. Тогда

$$
|\bar{x}(t)-z(t)| \leqslant c_{2}\left(\|\bar{u}\|^{2}+\|[\bar{v}]\|^{2}\right), \quad 0 \leqslant t \leqslant T,
$$

әде

$$
\begin{aligned}
& c_{2}=(1-L)^{-1} S \max \left\{2 c_{1}^{2}\left(K_{2} T+\sum_{i=1}^{p} L_{i}^{(2)}\right)+K_{3},\right. \\
&\left.2 c_{1}^{2}\left(K_{2} T+\sum_{i=1}^{p} L_{i}^{(2)}\right)+\max _{1 \leqslant i \leqslant p} L_{i}^{(3)}\right\} .
\end{aligned}
$$

Д о каз а т ель с т в о. Функция $\bar{x}(t)-z(t)$ является решением следующего интегрального уравнения:

$$
\begin{gathered}
\bar{x}(t)-z(t)=\int_{0}^{T} K(t, \tau) f_{x}(\tau, x(\tau), u(\tau))(\bar{x}(\tau)-z(\tau)) d \tau+ \\
+\sum_{i=1}^{p} K\left(t, t_{i}\right) I_{i x}\left(x\left(t_{i}\right), v_{i}\right)\left(\bar{x}\left(t_{i}\right)-z\left(t_{i}\right)\right)+ \\
+\sum_{i=1}^{p} K\left(t, t_{i}\right)\left[I_{i}\left(x\left(t_{i}\right)+\bar{x}\left(t_{i}\right), v_{i}+\bar{v}_{i}\right)-\right. \\
\left.-I_{i}\left(x\left(t_{i}\right), v_{i}\right)-I_{i x}\left(x\left(t_{i}\right), v_{i}\right) \bar{x}\left(t_{i}\right)-I_{i v_{i}}\left(x\left(t_{i}\right), v_{i}\right) \bar{v}_{i}\right]+ \\
+\int_{0}^{T} K(t, \tau)[f(\tau, x(\tau)+\bar{x}(\tau), u(\tau)+\bar{u}(\tau)-f(\tau, x(\tau), u(\tau))- \\
\left.\quad-f_{x}(\tau, x(\tau), u(\tau)) \bar{x}(\tau)-f_{u}(\tau, x(\tau), u(\tau)) \bar{u}(\tau)\right] d \tau .
\end{gathered}
$$


Учитывая условия 2), 5) и 7), устанавливаем следующую оценку:

$$
|\bar{x}(t)-z(t)| \leqslant(1-L)^{-1} S\left[\int_{0}^{T}\left(K_{2}|\bar{x}|^{2}+K_{3}|\bar{u}|^{2}\right) d t+\sum_{i=1}^{p}\left(L_{i}^{(2)}\left|\bar{x}\left(t_{i}\right)\right|^{2}+L_{i}^{(3)}\left|\bar{v}_{i}\right|^{2}\right)\right] .
$$

Наконец, учитывая утверждение леммы 1, получаем

$$
\begin{aligned}
|\bar{x}(t)-z(t)| \leqslant(1-L)^{-1} S\left[c_{1}^{2}\left(K_{2} T+\sum_{i=1}^{p} L_{i}^{(2)}\right)(\|\bar{u}\|+\|[\bar{v}]\|)^{2}+\right. & \\
& \left.+K_{3}\|\bar{u}\|^{2}+\sum_{i=1}^{p} L_{i}^{(3)}\left|\bar{v}_{i}\right|^{2}\right] .
\end{aligned}
$$

Так как $(a+b)^{2} \leqslant 2\left(a^{2}+b^{2}\right)$, из последнего получаем неравенство $(12)$.

ТЕОрема 3. Пусть выполняются условия 1)-8), кроме того,

$$
\operatorname{det}\left(E+I_{i x}\left(x\left(t_{i}\right), v_{i}\right)\right) \neq 0, \quad i=1,2, \ldots, p \text {. }
$$

Тогда функиионал (6) при ограничениях (2)-(5) дифберенцируем, причём его градиент имеет вид

$$
J^{\prime}(u,[v])=\left(f_{u}^{\prime}(t, x, u) \psi(t), \sum_{i=1}^{p} I_{i v_{i}}^{\prime}\left(x_{i}, v_{i}\right) \psi\left(t_{i}\right)\right) \in L_{2}^{r}[0, T] \times \mathbb{R}^{m}
$$

где $\psi(t)$ - решение дифференииально-разностной системь

$$
\begin{gathered}
\frac{d \psi(t)}{d t}=-f_{x}^{\prime}(t, x, u) \psi(t), \quad t \neq t_{i}, \\
\Delta \psi\left(t_{i}\right)=-I_{i x}^{\prime}\left(x\left(t_{i}\right), v_{i}\right)\left(I_{i x}^{\prime}\left(x\left(t_{i}\right), v_{i}\right)+E\right)^{-1} \psi\left(t_{i}\right), \quad i=1,2, \ldots, p,
\end{gathered}
$$

с граничными условиями

$$
\begin{aligned}
& A^{\prime}\left(A^{\prime}+B^{\prime}\right)^{-1} \psi(T)+B^{\prime}\left(A^{\prime}+B^{\prime}\right)^{-1} \psi(0)= \\
& \quad=B^{\prime}\left(A^{\prime}+B^{\prime}\right)^{-1} \Phi_{x(0)}(x(0), x(T))-A^{\prime}\left(A^{\prime}+B^{\prime}\right)^{-1} \Phi_{x(T)}(x(0), x(T)) .
\end{aligned}
$$

Доказательство. Пусть $(u,[v]),(u+\bar{u},[v+\bar{v}]) \in U \times \Pi^{p}-$ два допустимых управления. Тогда для приращения функционала (6) справедлива формула

$$
\begin{aligned}
& J(u+\bar{u},[v+\bar{v}])-J(u,[v])= \\
& \quad=\left\langle\Phi_{x(0)}(x(0), x(T)), z(0)\right\rangle+\left\langle\Phi_{x(T)}(x(0), x(T)), z(T)\right\rangle+\eta
\end{aligned}
$$

где

$$
\eta=\left\langle\Phi_{x(0)}(x(0), x(t)), \bar{x}(0)-z(0)\right\rangle+\left\langle\Phi_{x(t)}(x(0), x(t)), \bar{x}(t)-z(t)\right\rangle+
$$




$$
\begin{aligned}
+ & \Phi(x(0)+\bar{x}(0), x(t)+\bar{x}(t))-\Phi(x(0), x(t))- \\
& -\left\langle\Phi_{x(0)}(x(0), x(t)), \bar{x}(0)\right\rangle-\left\langle\Phi_{x(t)}(x(0), x(t)), \bar{x}(t)\right\rangle .
\end{aligned}
$$

K формуле (16) добавим нулевые слагаемые

$$
\int_{0}^{T}\left\langle\psi(t),-\frac{d z}{d t}+f_{x}(t, x(t), u(t)) z(t)+f_{u}(t, x(t), u(t)) \bar{u}(t)\right\rangle d t, \quad\langle\lambda, A z(0)+B z(T)\rangle,
$$

где $\psi(t) \in L_{2}^{r}[0, T]$ - пока произвольная функция, а $\lambda \in \mathbb{R}^{n}-$ произвольный числовой вектор.

После несложных преобразований для приращения функционала получаем следующую формулу:

$$
\begin{aligned}
& J(u+\bar{u},[v+\bar{v}])-J(u,[v])= \\
& =\sum_{i=0}^{p} \int_{t_{i}}^{t_{i+1}}\left\langle\dot{\psi}(t)+H_{x}(t, x(t), u(t), \psi(t)), z(t)\right\rangle d t+ \\
& +\int_{0}^{T}\left\langle H_{u}(t, x(t), u(t), \psi(t)), \bar{u}(t)\right\rangle d t+\sum_{i=1}^{p}\left\langle h_{i v_{i}}\left(x_{i}, v_{i}\right), \bar{v}_{i}\right\rangle+ \\
& \quad+\left\langle\Phi_{x(0)}(x(0), x(T))+A^{\prime} \lambda+\psi(0), z(0)\right\rangle+ \\
& \quad+\left\langle\Phi_{x(T)}(x(0), x(T))+B^{\prime} \lambda-\psi(T), z(T)\right\rangle+ \\
& +\left\langle\Delta \psi\left(t_{i}\right)+I_{i x}^{\prime}\left(x\left(t_{i}\right), v_{i}\right)\left(I_{i x}^{\prime}\left(x\left(t_{i}\right), v_{i}\right)+E\right)^{-1} \psi\left(t_{i}\right), z\left(t_{i}\right)\right\rangle+\eta
\end{aligned}
$$

где $H(t, x, u, \psi)=\langle\psi, f(t, x, u)\rangle, h_{i}\left(x_{i}, v_{i}\right)=\left\langle\psi\left(t_{i}\right), I_{i}\left(x_{i}, v_{i}\right)\right\rangle$. Теперь произвольную функцию $\psi(t) \in L_{2}^{r}[0, T]$ выбираем как решение дифференциальноразностного уравнения

$$
\begin{gathered}
\dot{\psi}(t)=-H_{x}(t, x(t), u(t), \psi(t)), \quad t \neq t_{i} \\
\Delta \psi\left(t_{i}\right)=-I_{x}^{\prime}\left(x\left(t_{i}\right), v_{i}\right)\left(I_{x}^{\prime}\left(x\left(t_{i}\right), v_{i}\right)+E\right)^{-1} \psi\left(t_{i}\right), \quad i=1,2, \ldots, p,
\end{gathered}
$$

которое совпадает с (13), (14), а числовой вектор $\lambda \in \mathbb{R}^{n}$ определяем из соотношений

$$
\begin{gathered}
\Phi_{x(T)}(x(0), x(T))+B^{\prime} \lambda-\psi(T)=0, \\
\Phi_{x(0)}(x(0), x(T))+A^{\prime} \lambda+\psi(0)=0 .
\end{gathered}
$$

ЗАмечание 2. Так как в краевых условиях (18), (19) присутствует векторный параметр $\lambda \in \mathbb{R}^{n}$, система уравнений (13), (14), (18), (19) называется сопряжённой системой в параметрическом виде.

Здесь, учитывая условие 3), можно исключить неизвестный вектор $\lambda \in \mathbb{R}^{n}$. Действительно, из (18),(19) имеем

$$
\lambda=\left(A^{\prime}+B^{\prime}\right)^{-1}\left[\psi(T)-\psi(0)-\Phi_{x(T)}(x(0), x(T))-\Phi_{x(0)}(x(0), x(T))\right] .
$$


Найденное значение учтём в (18) или (19), и после несложных преобразований получаем (15). Из равенства (17) получаем оценку

$$
\begin{aligned}
|\eta| \leqslant\left|\Phi_{x(0)}(x(0), x(T))\right||\bar{x}(0)-z(0)|+\left|\Phi_{x(T)}(x(0), x(T))\right||\bar{x}(T)-z(T)|+ \\
+\mid \Phi(x(0)+\bar{x}(0), x(T)+\bar{x}(T))-\Phi(x(0), x(T))- \\
-\left\langle\Phi_{x(0)}(x(0), x(T)), \bar{x}(0)\right\rangle-\left\langle\Phi_{x(T)}(x(0), x(T)), \bar{x}(T)\right\rangle \mid .
\end{aligned}
$$

Используя условия 8) и леммы 1 и 2, из последнего неравенства имеем

$$
|\eta| \leqslant\left[\left(K_{4}+K_{5}\right) c_{2}+c_{1}^{2}\left(K_{7}+K_{6}\right)\right]\left(\|\bar{u}\|^{2}+\|[\bar{v}]\|^{2}\right) .
$$

ЗАмечание 3. Если матрицы $A$ и $B$ коммутативны, т. е. $A B=B A$, то граничные условия (15) сопряжённой системы упрощаются:

$$
A^{\prime} \psi(T)+B^{\prime} \psi(0)=B^{\prime} \Phi_{x(0)}(x(0), x(T))-A^{\prime} \Phi_{x(T)}(x(0), x(T)) .
$$

4. Необходимые условия оптимальности. Имея формулы градиента для функционала (6) при ограничениях (2)-(5), можно получить необходимые условия оптимальности в задаче оптимального управления.

Теорема 4. Пусть выполнены условия теоремы 3. Тогда для оптимальности управления $\left(u_{*},[v]_{*}\right) \in U \times \Pi^{p}$ в задаче $(2)-(6)$ необходимо выполнение неравенства

$$
\int_{0}^{T}\left\langle H_{u}\left(t, x_{*}(t), u_{*}(t), \psi_{*}(t)\right), u(t)-u_{*}(t)\right\rangle d t+\sum_{i=1}^{p}\left\langle h_{i v_{i}}\left(x_{i *}, v_{i *}\right), v_{i}-v_{i *}\right\rangle \geqslant 0
$$

для любого $(u,[v]) \in U \times \Pi^{p}$, где $x_{*}(t)=x\left(t ; u_{*},[v]_{*}\right), \psi_{*}(t)=\psi\left(t ; u_{*},[v]_{*}\right)$.

Доказ ательство. Множество $U \times \Pi^{p}$, определяемое равенством (5), выпукло в пространстве $L_{2}[0, T] \times \Pi^{p}$. Кроме того, согласно теореме 3, функционал $J(u,[v])$ дифференцируем по Фреше на множестве $U \times \Pi^{p}$. Тогда в силу теоремы 3 из $\left[10\right.$, с. 524] на элементе $\left(u_{*},[v]_{*}\right) \in U \times \Pi^{p}$ необходимо выполнение неравенства $\left\langle J^{\prime}\left(u_{*},[v]_{*}\right),(u,[v])-\left(u_{*},[v]_{*}\right)\right\rangle \geqslant 0$ при всех $(u,[v]) \in U \times \Pi^{p}$. Отсюда и из (13) следует справедливость неравенства (20).

Из теоремы 4 следует очевидное

СледствиЕ. Пусть выполнены условия теоремы 3. Тогда для оптимальности управления $\left(u_{*},[v]_{*}\right) \in U \times \Pi^{p}$ в задаче $(2)-(6)$ необходимо выполнение неравенств

$$
\begin{gathered}
\int_{0}^{T}\left\langle H_{u}\left(t, x_{*}(t), u_{*}(t), \psi_{*}(t)\right), u(t)-u_{*}(t)\right\rangle d t \geqslant 0 \\
\sum_{i=1}^{p}\left\langle h_{i v_{i}}\left(x_{i *}, v_{i *}\right), v_{i}-v_{i *}\right\rangle \geqslant 0
\end{gathered}
$$

для любого $(u,[v]) \in U \times \Pi^{p}$, где $x_{*}(t)=x\left(t ; u_{*},[v]_{*}\right), \psi_{*}(t)=\psi\left(t ; u_{*},[v]_{*}\right)$. 
Заключение. Теорема 1 носит вспомогательный характер и позволяет нелокальную краевую задачу представить в виде интегрального уравнения, которое, в свою очередь, упрощает исследование существования и единственности решений исходной краевой задачи. Теорема 2 даёт достаточное условие существования и единственности решения краевой задачи (2)-(4) при каждом фиксированном допустимом управлении. Отметим, что из схемы доказательств теорем видно, что данную схему можно успешно применять для более сложных задач оптимального управления с нелокальными условиями при импульсных воздействиях.

\section{БИБЛИОГРАФИЧЕСКИЙ СПИСОК}

1. А. М. Самойленко, Н. А. Перестюк, Дифференциальные уравнения с импульсным воздействием. Киев: Вища школа, 1987. 287 с.; англ. пер.: A. M. Samoilenko, N. A. Perestyuk, Impulsive Differential Equations. Singapore: World Scientific, 1995. $\mathrm{ix}+467 \mathrm{pp}$.

2. A. Halanay, D. Wexler, Teoria calitativă a sistemelor cu impulsuri (Qualitative Theory of Sampled-Data Systems). Bucuresti: Editura Academiei Republicii Socialiste România, 1968. 312 pр.; русск. пер.: А. Халанай, Д. Векслер, Качественная теория импульсных систем. Москва: Мир, 1971. 309 с.

3. V. Lakshmikantham, D. D. Bainov, P. S. Simeonov, Theory of impulsive differential equations / Series in Modern Applied Mathematics. Vol. 6. World Scientific: Singapore, 1989. xii+273 pp.

4. M. Benchohra, J. Henderson, S. Ntouyas, Impulsive differential equations and inclusions / Contemporary Mathematics and Its Applications. Vol. 2. Hindawi Publishing Corporation: New York, 2006. xiv+366 pp.

5. B. Selvaraj, M. Mallika Arjunan, V. Kavitha, "Existence of solutions for impulsive nonlinear differential equations with nonlocal conditions" // J. KSIAM, 2009. Vol. 13, no. 3. Pp. 203215, http://mathnet.kaist.ac.kr/mathnet/thesis_file/jksiam-v13n3p203.pdf.

6. A. Anguraj, M. Mallika Arjunan, "Existence and uniqueness of mild and classical solutions of impulsive evolution equations"// Electron. J. Differential Equations, 2005. Vol. 2005, no.111. Pp. 1-8, http://www.kurims.kyoto-u.ac.jp/EMIS/journals/EJDE/ Volumes/2005/111/anguraj ·pdf.

7. S. Ji, S. Wen, "Nonlocal Cauchy problem for impulsive differential equations in Banach spaces" // Int. J. Nonlinear Sci, 2010. Vol. 10, no. 1. Pp. 88-95.

8. M. Li, M. Han, "Existence for neutral impulsive functional differential equations with nonlocal conditions" // Indag. Math. (N.S.), 2009. Vol. 20, no. 3. Pp. 435-451.

9. А. М. Самойленко, Н. И. Ронто, Численно-аналитические методы исследования решений краевых задач. Киев: Наукова думка, 1986. 223 с. [A. M. Samoylenko, N.I. Ronto, Numerical-analytic methods for investigating the solutions of boundary value problems. Kiev: Naukova Dumka, 1986. 223 pp.]

10. Ф. П. Василъев, Методы оптимизации. Москва: Факториал Пресс, 2002. 823 с. [F. P. Vasilyev, Optimization methods. Moscow: Factorial Press, 2002. 823 pp.]

Поступила в редакцию 09/XI/2012;

в окончательном варианте - 21/IX/2013. 
MSC: 34B37; 49K15

\title{
OPTIMAL CONTROL PROBLEM FOR THE IMPULSIVE DIFFERENTIAL EQUATIONS WITH NON-LOCAL BOUNDARY CONDITIONS
}

\author{
Ya. A. Sharifov
}

1 Baku State University,

23, Z. Khalilov st., Baku, AZ-1073/1, Azerbaijan.

2 Institute of Cybernetics named after Academician A. Huseynov,

National Academy of Sciences of Aserbaijan,

9, F. Agayev st., Baku, AZ1141, Azerbaijan.

E-mail: sharifov22@rambler.ru

The optimal control problem is investigated, where the state of the controlled system is described by the impulsive differential equations with non-local boundary conditions. The existence and uniqueness of the non-local impulsive boundary problem by fixed admissible controls are proved using the contraction mapping principle. The gradient of the functional is calculated under certain conditions on the initial data. The necessary conditions for optimality of the first order are obtained.

Keywords: non-local boundary conditions, impulsive systems, necessary conditions of optimality, gradient of functional, existence and uniqueness of the solution.

Original article submitted 09/XI/2012; revision submitted 21/IX/2013.

Yagub A. Sharifov (Ph.D. Phys. \& Math.), Associate profesor, Dept. of Mathematical Methods of Applied Analysis ${ }^{1}$; Senior Researcher, Lab. of Numerical Methods of Optimal Control $^{2}$. 